\title{
TRIGO DURO: COMPORTAMENTO DE GENÓTIPOS NO ESTADO DE SÃO PAULO $\left({ }^{1}\right)$
}

\author{
CARLOS EDUARDO DE OLIVEIRA CAMARGO $\left({ }^{2.4}\right)$, \\ JOÃO CARLOS FELÍCIO $\left({ }^{2}\right)$, RUI RIBEIRO DOS SANTOS $\left(^{3}\right)$ \\ e ANTONIO WILSON PENTEADO FERREIRA FILHO $\left({ }^{2}\right)$
}

\begin{abstract}
RESUMO
Compararam-se 25 linhagens de trigo duro (Triticum durum L.), um cultivar de triticale (X Triticosecale Wittmack) e quatro cultivares de trigo ( $T$. aestivum L.) em seis experimentos em condição de irrigação por aspersão, analisando-se a produção de grãos, características agronômicas e resistência às doenças. As linhagens de trigo duro 14 (61150/Leeds//Gallo "S"/3/Garza "S"/4/Mexicali "S"/5/S-15-Crane "S"), 21 (Boyeros "S"/Cocorit-71/5/Crane "S"/Ganso "S"//Marte "S"/3/Tildillo "S"/4/Memo "S"), 25 (Gallareta "S") e 8 (Gediz "S"/Yavaros "S"), de porte baixo a médio, foram resistentes às ferrugens-do-colmo (com exceção da 21) e da-folha, moderadamente resistentes ao oídio, suscetíveis à mancha foliar, e destacaram-se quanto à produção de grãos em solos com baixa acidez, não diferindo nem do trigo comum IAC-60, o mais cultivado atualmente no Estado de São Paulo, nem do triticale Álamos. Em condições de campo, a linhagem de trigo duro 19 (Mindum/Kingfisher "S"//Sandpiper) apresentou imunidade às ferrugens-do-colmo e da-folha e foi moderadamente resistente ao oídio. O triticale Álamos e o trigo comum IAC-29 foram imunes ao oídio. Todos os genótipos avaliados foram altamente suscetíveis à mancha foliar, com exceção da linhagem 6 (Dackiye/Gerardo Vezio 394), moderadamente resistente.
\end{abstract}

Termos de indexação: trigo duro, Triticum durum L., trigo, $T$. aestivum L.; triticale $\mathrm{X}$ Triticosecale Wittmack, linhagens, cultivares, produção de grãos, características agronômicas, resistência às doenças.

(') Com recursos suplementares do Acordo do Trigo entre as Cooperativas Rurais do Vale do Paranapanema e a Secretaria de Agricultura e Abastecimento, através do Instituto Agronômico. Recebido para publicação em 9 de setembro de 1994 e aceito em 3 de agosto de 1995.

(2) Seção de Arroz e Cereais de Inverno, Instituto Agronômico (IAC), Caixa Postal 28, $13001-970$ Campinas (SP).

$\left({ }^{3}\right)$ Estação Experimental de Monte Alegre do Sul, IAC.

(4) Com bolsa de pesquisa do CNPq. 


\title{
ABSTRACT \\ DURUM WHEAT: EVALUATION OF GENOTYPES FOR THE STATE OF SÃO PAULO, BRAZIL
}

\begin{abstract}
Twenty-five durum wheat (Triticum durum L.) lines, one triticale (X Triticosecale Wittmack) cultivar and four wheat ( $T$. aestivum $\mathrm{L}$.) cultivars were evaluated in six trials under sprinkler irrigation taking into account the grain yield, agronomic characteristics and disease resistance. The durum wheat lines $14(61150 /$ Leeds//Gallo "S"/3/Garza "S"/4/Mexicali "S"/5/S-15-Crane "S"), 21 (Boyeros "S"/Cocorit-71/5/Crane "S"/Ganso "S"//Marte "S"/3/Tildillo "S"/4/Memo "S"), 25 (Gallareta "S") and 8 (Gediz "S"/Yavaros "S"), showed the following traites: resistant to stem and leaf rusts; moderately resistant to powdery mildew; susceptible to leaf spot, and short to medium stature. These lines presented good grain yield in soils with low acidity. However, they did not differ in yield from the wheat cultivar IAC-60, the most cultivated actually in the State of São Paulo and from the triticale Álamos. The durum wheat line 19 (Mindum/Kingfisher "S"//Sandpiper) showed at the same time immunity to the causal agents of stem (with exception of line 21) and leaf rusts and moderate resistance to the causal agent of powdery mildew. The cultivars Álamos (triticale) and IAC-29 (wheat) cxhibited immunity to the causal agents of powdery mildew. All considered genotypes were susceptible to the leaf spots patogens, with exception of the durum wheat line 6 (Dackiye/Gerardo Vezio 394) which was moderately resistant.
\end{abstract}

Index terms: durum wheat, Triticum durum L., trigo, T. aestivum L., triticale, $\mathrm{X}$ Triticosecale Wittmack, inbred lines, cultivars, grain yield, agronomic characteristics, disease resistance.

\section{INTRODUÇÃO}

Para atender à demanda da indústria de pastas alimentares, alimento de grande consumo entre a população brasileira de baixa renda, é que se iniciaram, em 1984, os trabalhos de melhoramento de trigo duro (Triticum durum L.) no Instituto Agronômico (São Paulo, 1984-1992). Esse projeto de pesquisa tem introduzido e avaliado, anualmente, coleções e ensaios de variedades dessa espécie que mostra 28 cromossomos ( $2 n$ ) em comparação com o trigo comum (T. aestivum L.) que apresenta 48 .

Estudaram-se, conjuntamente, linhagens de trigo duro, cultivares de trigo comum e de triticale tanto em solo ácido $\left(\mathrm{V} \%=14 \mathrm{e} \mathrm{H}^{+}+\mathrm{Al}^{3+}=89 \mathrm{mmol}_{\mathrm{c}} / \mathrm{dm}^{3}\right)$ como em solo corrigido $\left(\mathrm{V} \%=65 \mathrm{e} \mathrm{H}^{+}+\mathrm{Al}^{3+}=29\right.$ $\mathrm{mmol}_{\mathrm{c}} / \mathrm{dm}^{3}$ ). As produções de trigo duro em solo ácido foram baixas, variando de 939 a $2.243 \mathrm{~kg} / \mathrm{ha}$, enquanto as de trigo comum e as de triticale, tolerantes ao $\mathrm{Al}^{3+}$, variaram de 3.584 a $4.922 \mathrm{~kg} / \mathrm{ha}$. Em solo corrigido, a melhor linhagem de trigo duro
(Avetoro "S" x Anhinga "S" - Pelicano "S" x D. 67.2) produziu $4.128 \mathrm{~kg} / \mathrm{ha}$, em comparação ao triticale (Chiva), com $4.545 \mathrm{~kg} / \mathrm{ha}$, e ao melhor trigo, IAC-24, com $4.906 \mathrm{~kg} / \mathrm{ha}$ (Camargo et al., 1992).

Em outros experimentos, realizados em solos corrigidos e com irrigação por aspersão, destacaram-se as linhagens de trigo duro (Avctoro " $\mathrm{S}$ " $\mathrm{x}$ Anhinga "S" - Pelicano "S" x D.67.2), Gallareta "S" e Yavaros "S", não diferindo dos genótipos de trigo e triticale mais produtivos (Camargo et al., 1995).

Empregando soluções nutritivas, Camargo et al. (1993) verificaram que 25 linhagens de trigo duro foram altamente sensíveis à toxicidade de $\mathrm{Al}^{3+}$. Confirmou-se, desse modo, a necessidade de incorporar tolerância ao $\mathrm{Al}^{3+}$ nos genótipos de trigo duro visando ao cultivo em solos ácidos.

As farinhas obtidas das linhagens de trigo duro Yavaros "S", Tel1-76, (Avetoro "S" x Anhinga "S" - Pelicano "S"/Flamingo "S"), (Dackiye - Rabi- 
corno "S" x Yavaros "S") e (Stifftail "S" x Yavaros "S") mostraram qualidades tecnológicas adequadas para a produção de macarrão, enquanto as dos trigos comuns IAC-24 e IAC-60 se apresentaram adequadas à produção de pães (Camargo et al., 1993).

Considerando os resultados de pesquisa já obtidos no Estado de São Paulo e a instalação de moinhos especiais no Brasil para a produção de farinhas de trigo duro (semolina), inicialmente moendo grãos importados e, ainda, que as pastas alimentícias produzidas com essa matéria-prima apresentam maior valor nutritivo aliado à estabilidade no cozimento, isto é, não tendem a desintegrar, a apresentar gomosidade na fervura, nem a tornar-se moles e pastosas quando mantidas na água após o cozimento e que, finalmente, no mercado internacional, os trigos duros de boa qualidade geralmente alcançam preços mais altos que os de panificação (Hanson et al., 1982), seria de grande importância a implantação da cultura de trigo duro no País como alternativa para a agricultura brasileira no inverno.

Este trabalho teve por objetivo avaliar 25 linhagens de trigo duro em comparação com quatro cultivares de trigo comum e um de triticale em condições de irrigação por aspersão em Monte Alegre do Sul e Campinas, quanto à produção de grãos, altura das plantas, ciclo da emergência ao florescimento, acamamento e resistência às doenças, visando à escolha das mais promissoras para multiplicação e posterior lançamento aos agricultores.

\section{MATERIAL E MÉTODOS}

\subsection{Genealogia dos genótipos estudados}

A genealogia e a relação das 25 linhagens de trigo duro, introduzidas do Centro Internacional de Melhoramento de Milho e Trigo (CIMMYT), México, encontram-se a seguir:

$\begin{array}{ll}\text { 1. } & \text { Rockel "S" } \\ 2 . & \text { Boyeros "S" } \\ \text { 3. } & \text { Tringa "S" } \\ \text { 4. } & \text { Swan "S" }\end{array}$
5. Scoter "S"/Mexicali "S"
6. Dackiye/Gerardo Vezio 394
7. Boyeros "S"/Yavaros "S"
8. Gediz "S"/Yavaros "S"
9. Carcomum "S"
10, 11 e 12. Memo "S"/Mexicali 75
13. Memo "S"/Goose "S"
14. 61150/Leeds//Gallo "S"/3/Garza "S"/4/Mexicali "S"/5/S-15-Crane "S"
15. USDA $580 / 3 /$ Cocorit "S"/Sacaba $81 / /$ Flamingo "S"/4/Goose "S"
16. USDA 595/Dackiye "S"//Rokel "S"
17. Ganso "S"/Tehuacan 60//Mexicali "S"/3/Gediz "S"/4/Celta "S"
$18 . \quad$ Rail "S"
19. Mindum/Kingfisher "S"//Sandpiper
20. Scoter/Rabicorno "S"//Mexicali "S"
21. Boyeros "S"/Cocorit-71/5/Crane "S"/Ganso "S"//Marte "S"/3/Tildillo "S"/4/Memo "S"
22.
Gaviota "S"/Durum 69
23. Mexicali 75
24. Yavaros 79
25. Gallareta "S"

Como controles, utilizaram-se o cultivar de triticale Álamos (T-26) e os de trigo: Seri-82 (T-27), IAC-24 (T-28), IAC-60 (T-29) e IAC-29 (T-30), e cuja origem é a seguinte:

Álamos: selecionado no CIMMYT;

Seri-82: obtido por seleção no CIMMYT a partir do cruzamento Kavkaz/Buho "S"//Kaliansona/Bluebird;

IAC-24: Selecionado no IAC a partir do híbrido 'IAS-51' x 'IRN 597-70';

IAC-60: obtido por seleção a partir do cruzamento 'IRN 33-70' x IAC-5, no IAC;

IAC-29: provindo de seleção realizada no IAC e oriundo do cruzamento entre o híbrido IBN 157-72 $x$ IAC-5, seguido de um retrocruzamento para o cultivar IAC-5. 


\subsection{Ensaios de genótipos em condição de campo e de laboratório}

Utilizou-se o delineamento estatístico de blocos ao acaso com três repetições por local. Cada ensaio foi constituído de 90 parcelas, cada uma formada de seis linhas de $3 \mathrm{~m}$ de comprimento, espaçadas de $0,2 \mathrm{~m}$, e uma separação lateral entrc as parcelas de $0,6 \mathrm{~m}$. Fez-se a semeadura à base de 80 sementes viáveis por metro de sulco, equivalendo a 1.440 sementes por parcela, com uma área útil de colheita de $3,60 \mathrm{~m}^{2}$.

Efetuaram-se dois ensaios no Centro Experimental de Campinas em 1987-88, e quatro ensaios na Estação Experimental de Monte Alegre do Sul em 1986-89, todos com irrigação por aspersão. Os resultados da análise das amostras compostas dos solos dos locais estudados encontram-se no quadro 1 .

Coletaram-se os seguintes dados: ferrugem-do-colmo (Puccinia graminis f. sp. tritici); ferrugem-da-folha ( $P$. recondita); mancha-da-folha (Helminthosporium sp. e/ou Septoria sp.); oídio (Erysiphe graminis sp. tritici); ciclo da emergência ao florescimento; acamamento; altura das plantas e produção de grãos. Avaliaram-se esses parâmetros conforme Schramm et al. (1974), Mehta (1978) e Camargo et al. (1991, 1993).

Os dados de produção de grãos de cada experimento foram submetidos à análise da variância, utilizando-se o teste $\mathrm{F}$ ao nível de $5 \%$ para detectar efeitos significativos de genótipos e repetições. Analisaram-se as variâncias conjuntas para os experimentos de Monte Alegre do Sul e Campinas para produção de grãos para detectar, pelo teste $F$, ao nível de $5 \%$, as significâncias dos efeitos de experimentos, genótipos e interação genótipos $\mathrm{x}$ experimentos. Fez-se a comparação das produções médias dos genótipos em cada ensaio e em cada grupo de experimentos pelo teste de Tukey ao nível de 5\% (Pimentel Gomes, 1985).

Procedeu-se à análise conjunta da variância para a característica altura das plantas, considerando a média dessa característica em cada um dos experimentos, para detectar pelo teste $F$, ao nível de $5 \%$, as significâncias dos efeitos de genótipos e experimentos. A comparação das médias dos genótipos para essa característica foi feita também pelo teste de Tukey ao nível de 5\%.

Quadro 1. Análises das amostras compostas dos solos ${ }^{(1)}$ dos locais onde foram instalados os ensaios de linhagens de trigo duro (Triticum durum L.) em comparação com cultivares de trigo (Triticum aestivum L.) e de triticale (X Triticosecale Wittmack) em 1986-89

\begin{tabular}{lcccccc} 
& \multicolumn{3}{c}{ Monte Alegre do Sul } & & \multicolumn{2}{c}{ Campinas } \\
\cline { 2 - 3 } \cline { 6 - 7 } Determinações & 1986 & 1987 & 1988 & 1989 & 1987 & 1988 \\
\hline P resina $(\mathrm{mg} / \mathrm{kg})$ & 85 & 61 & 19 & 18 & 43 & 26 \\
$\mathrm{M} . \mathrm{O} .(\mathrm{g} / \mathrm{kg})$ & 26 & 20 & 24 & -- & 42 & 25 \\
$\mathrm{pH}\left(\mathrm{CaCl}_{2}\right)$ & 5,0 & 4,9 & 5,1 & 5,0 & 4,9 & 4,6 \\
$\mathrm{~K}^{+}\left(\mathrm{mmol}_{\mathrm{c}} / \mathrm{dm}^{3}\right)$ & 2,8 & 3,2 & 2,6 & 1,6 & 3,2 & 2,3 \\
$\mathrm{Ca}^{2+} "$ & 27 & 25 & 21 & 18 & 39 & 17 \\
$\mathrm{Mg}^{2+} "$ & 7 & 6 & 7 & 6 & 18 & 10 \\
$\mathrm{H}^{+}+\mathrm{Al}^{3+} "$ & 28 & 29 & 28 & 28 & 37 & 39 \\
$\mathrm{~S}$ & 37 & 34 & 31 & 26 & 60 & 29 \\
$\mathrm{~T}$ & 65 & 63 & 59 & 54 & 97 & 68 \\
$\mathrm{~V} \%$ & 57 & 54 & 53 & 48 & 62 & 43 \\
\hline
\end{tabular}

(') Análises efetuadas pela Seçāo de Fertilidade do Solo e Nutrição de Plantas, IAC. 


\section{RESULTADOS E DISCUSSÃO}

Os quadrados médios das análises individuais da variância das produções de grãos, genótipos de trigo duro, trigo e triticale estudados em Monte Alegre do Sul (1986-89) e Campinas (1987-88) encontram-se no quadro 2. Os dados mostraram efeitos não significativos para genótipos e para repctições somente nos ensaius de Monte Alegre do Sul (1988) e de Monte Alegre do Sul (1986) respectivamente.

Os quadrados médios da análise conjunta da variância das produções médias dos genótipos dos ensaios, em Monte Alegre do Sul, mostraram efeitos significativos para anos e interação genótipos $\mathrm{x}$ anos, porém os efeitos de genótipos foram não significativos - Quadro 3.

Através do teste de Tukey, aplicado ao nível de $5 \%$, para a comparação das médias de produção de grãos dos genótipos dos ensaios instalados em Monte Alegre do Sul - Quadro 4 - verificou-se, em 1986, que a linhagem de trigo duro 21 mostrou a maior produção de grãos $(5.311 \mathrm{~kg} / \mathrm{ha})$, diferindo, porém, somente das linhagens 2,9 e 20 ; no ensaio de 1987 , o cultivar de trigo Seri- 82 foi o mais produtivo, com $6.254 \mathrm{~kg} / \mathrm{ha}$, não diferindo somente das linhagens $2,4,8,14,16,21,23,24$ e 25 , dos cultivares IAC- 24 , IAC-60 e IAC-29 e do

Quadro 2. Quadrados médios das análises individuais da variância das produções de grãos, das linhagens de trigo duro (Triticum durum L.) em comparação com cultivares de trigo (Triticum aestivum L. e de triticale (X Triticosecale Wittmack), em ensaios desenvolvidos em condição de irrigação na Estação Experimental de Monte Alegre do Sul (1986-89) e no Centro Experimental de Campinas (1987-88)

\begin{tabular}{|c|c|c|c|c|c|c|c|}
\hline \multirow{2}{*}{$\begin{array}{c}\text { Causas } \\
\text { de variação }\end{array}$} & \multirow{2}{*}{ G.L. } & \multicolumn{4}{|c|}{ Monte Alegre do Sul } & \multicolumn{2}{|c|}{ Campinas } \\
\hline & & 1986 & 1987 & 1988 & 1989 & 1987 & 1988 \\
\hline Repetições & 2 & 519.813 & $1.598 .252^{*}$ & $2.002 .573 *$ & $790.469 *$ & $1.791 .180^{*}$ & $610.581 *$ \\
\hline Genótipos & 29 & $1.402 .917^{*}$ & $1.799 .475^{*}$ & 735.368 & $662.648 *$ & $898.342 *$ & $380.912 *$ \\
\hline Resíduos & 58 & 362.120 & 427.640 & 524.931 & 241.590 & 310.485 & 183.634 \\
\hline
\end{tabular}

* = Significativo ao nível de $5 \%$.

Quadro 3. Quadrados médios das análises conjuntas da variância para produção de grãos, das linhagens de trigo duro (Triticum durum L.) em comparação com cultivares de trigo (Triticum aestivum L.) e de triticale (X Triticosecale Wittmack) em ensaios realizados em condição de irrigação na Estação Experimental de Monte Alegre do Sul (1986-89) e no Centro Experimental de Campinas (1987-88)

\begin{tabular}{lrrrrrr} 
Causas de variação & G.L. $\left({ }^{1}\right)$ & Q.M. $\left({ }^{1}\right)$ & G.L. $\left({ }^{2}\right)$ & Q.M. $\left({ }^{2}\right)$ & G.L. $\left({ }^{3}\right)$ & Q.M. $\left({ }^{3}\right)$ \\
\hline Anos (experimentos) & 3 & $69.959 .017^{*}$ & 1 & $47.432 .666^{*}$ & 5 & $53.366 .158^{*}$ \\
Genótipos & 29 & 1.577 .827 & 29 & $957.183^{*}$ & 29 & $1.386 .389^{*}$ \\
Genótipos x anos (experimentos) & 87 & $1.007 .527^{*}$ & 29 & 325.520 & 145 & $898.655^{*}$ \\
Resíduo & 240 & 417.027 & 120 & 278.854 & 360 & 370.969 \\
\hline
\end{tabular}

* Significativo ao nível de 5\%. (') Referentes aos ensaios de Monte Alegre do Sul (1986-89). ( ${ }^{2}$ ) Relativos aos ensaios de Campinas (1987-89). ( $\left.{ }^{3}\right)$ Concernentes aos ensaios de Monte Alegre do Sul (1986-89) e Campinas (1987-88). 


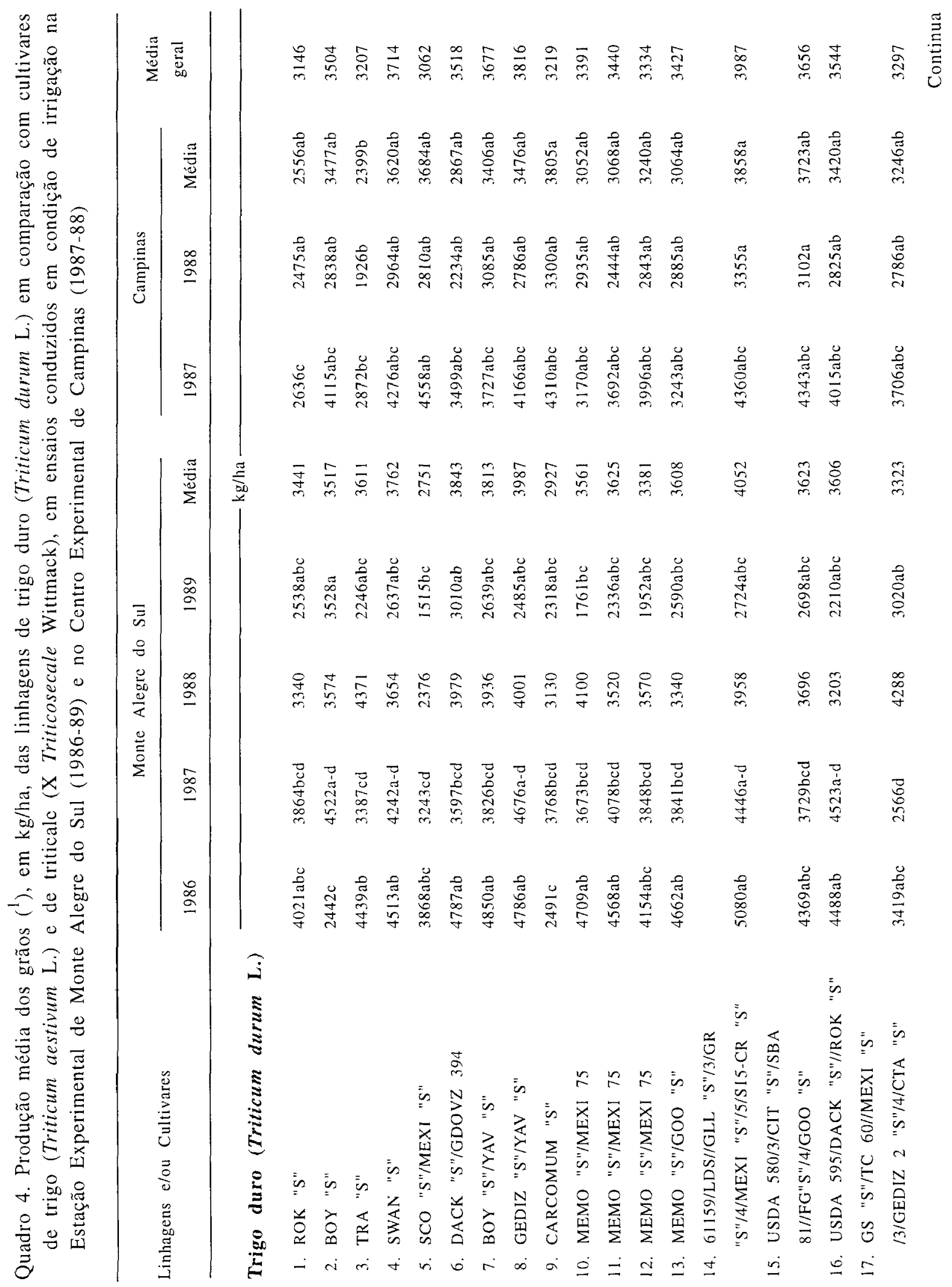




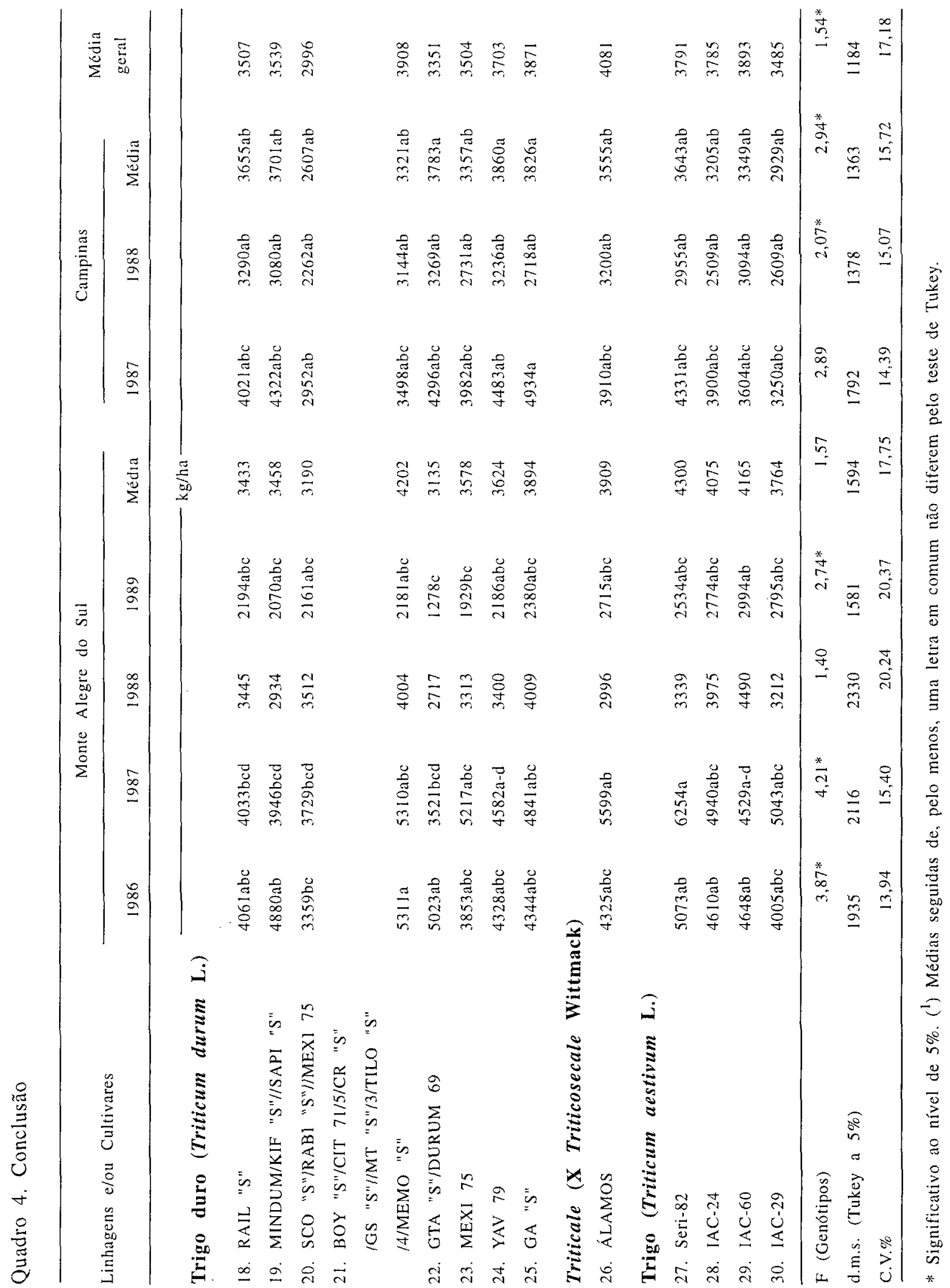




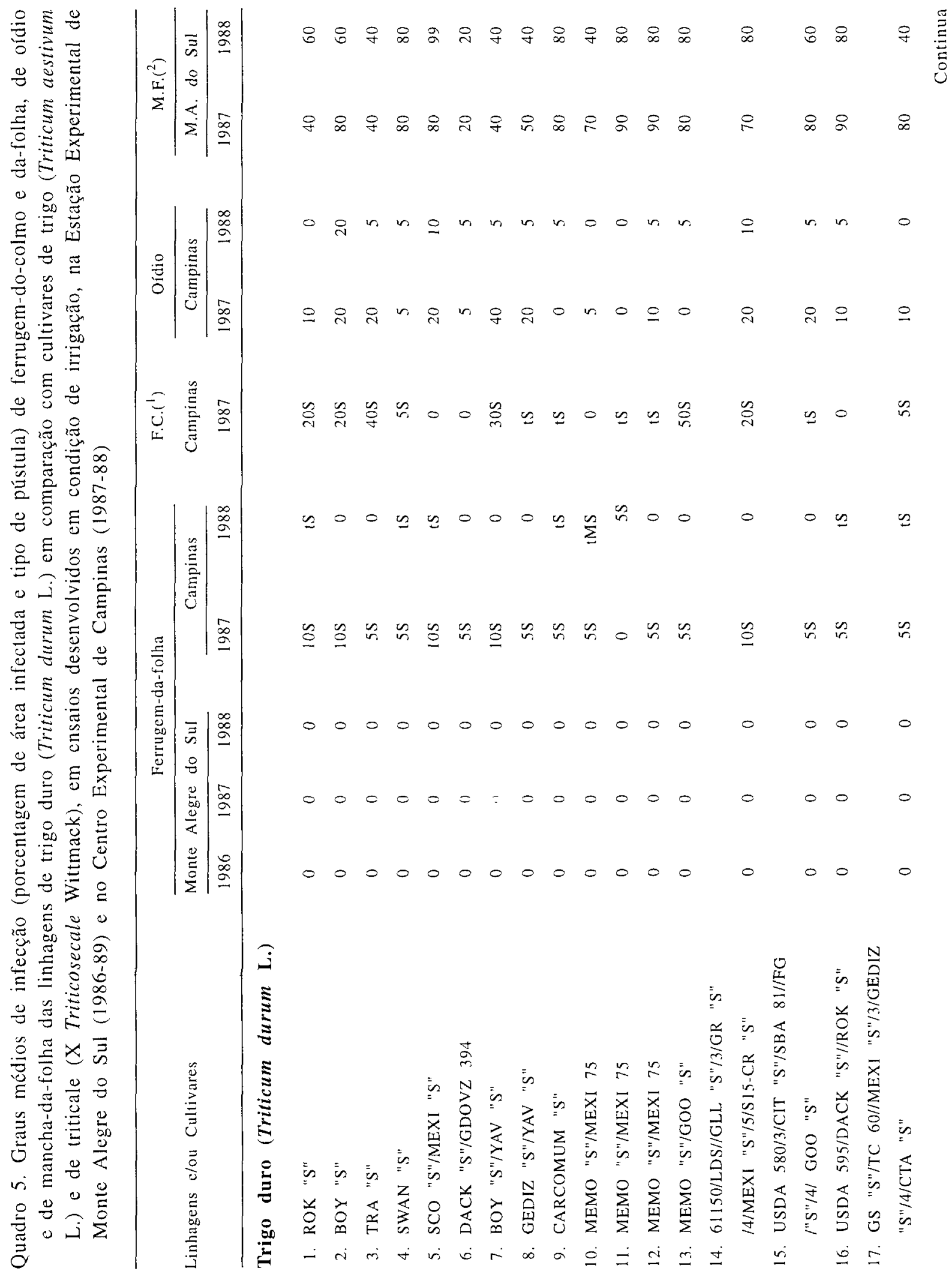




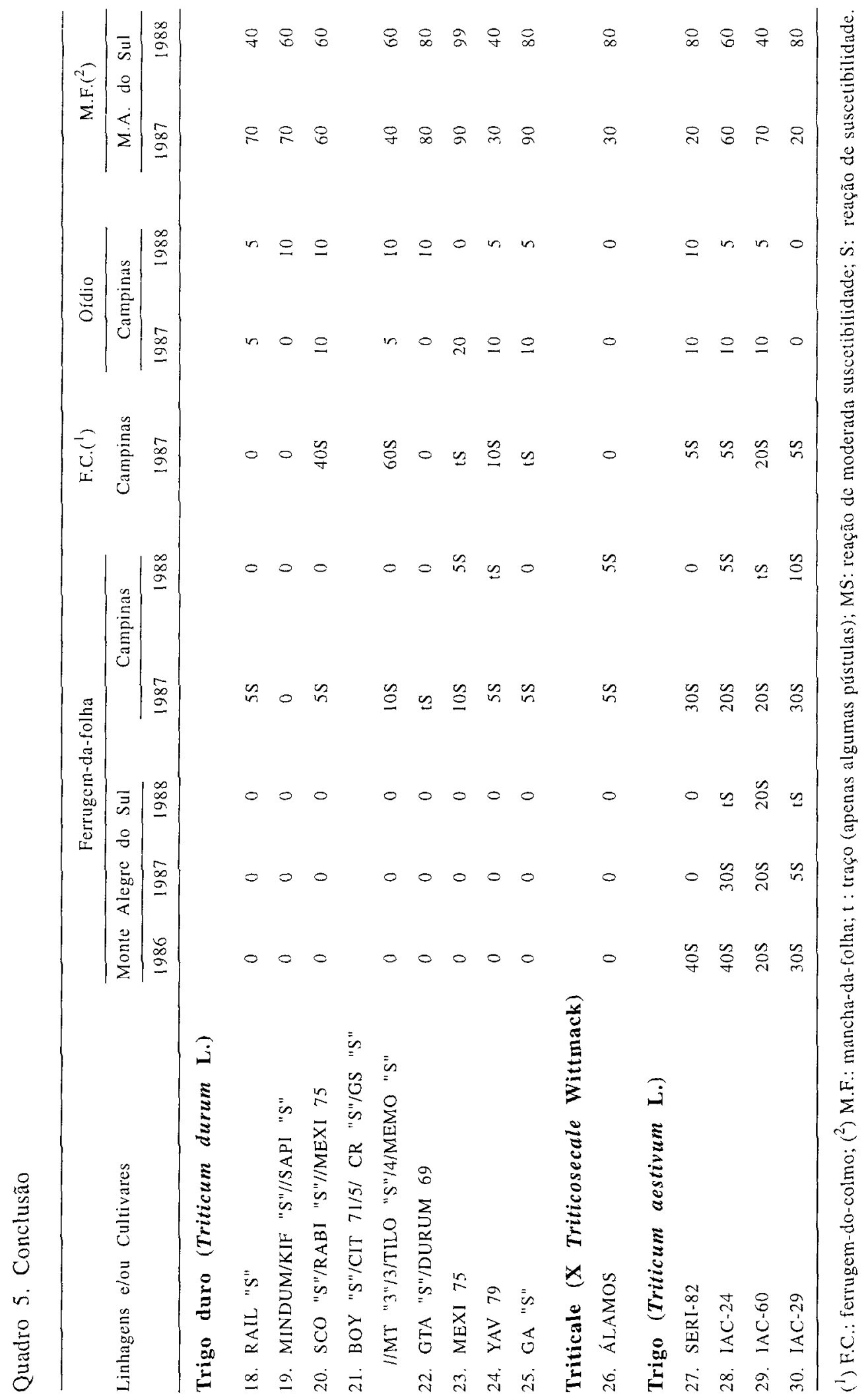


triticale Álamos; no de 1988, apesar de não haver diferença significativa entre os genótipos estudados, destacaram-se, pela produtividade, IAC- 60 $(4.490 \mathrm{~kg} / \mathrm{ha})$ e as linhagens 3 e 17 , que produziram 4.371 e $4.288 \mathrm{~kg} / \mathrm{ha}$ respectivamente; no ensaio de 1989 , a linhagem 2 mostrou a maior produção de grãos $(3.528 \mathrm{~kg} / \mathrm{ha})$, diferindo, porém, apenas das linhagens 5, 10, 22 e 23. Considerando a média dos quatro experimentos de Monte Alegre do Sul, observou-se que as linhagens de trigo duro 21 e 14 produziram 4.202 e $4.052 \mathrm{~kg} / \mathrm{ha}$ respectivamente, enquanto os cultivares de trigo Seri-82, IAC-60 e IAC-24 e o cultivar de triticale Álamos produziram $4.300,4.165,4.075$ e $3.909 \mathrm{~kg} / \mathrm{ha}$ respectivamente; contudo, não foram observadas diferenças mínimas significativas entre os genótipos avaliados.

Os quadrados médios da análise conjunta da variância das produções médias de grãos dos genótipos estudados nos ensaios instalados em Campinas (1987-88) mostraram efeitos significativos para anos e genótipos e não significativo para a interação genótipos $x$ anos - Quadro 3.

As produções médias de grãos dos genótipos dos ensaios de Campinas (1987-88) encontram-se no quadro 4. No ensaio de 1987, a linhagem de trigo duro 25 mostrou a maior produção de grãos $(4.934 \mathrm{~kg} / \mathrm{ha})$, diferindo, porém, somente das linhagens 1, 3 e 20; em 1988, a linhagem de trigo duro 14 foi a mais produtiva $(3.355 \mathrm{~kg} / \mathrm{ha})$, diferindo, porém, apenas da linhagem 3 . Considerando a média dos dois ensaios de Campinas (Quadro 4), as linhagens $24,14,25,9$ e 22 apresentaram maior produção de grãos: $3.860,3.858,3.826,3.805$ e 3.783 $\mathrm{kg} / \mathrm{ha}$ respectivamente, diferindo, apenas, da linhagem 3.

Os quadrados médios da análise conjunta da variância das produções médias de grãos dos genótipos estudados nos ensaios realizados em Monte Alegre do Sul e Campinas mostraram efeitos significativos para experimentos, genótipos e interação genótipos x experimentos - Quadro 3.

Considerando as médias de produções de grãos dos genótipos nos seis experimentos - Quadro 4 verificou-se não haver diferenças significativas entre eles pelo teste de Tukey, aplicado ao nível de 5\%; contudo, sobressaíram-se pela produtividade o cultivar de triticale Álamos $(4.081 \mathrm{~kg} / \mathrm{ha})$, o de trigo IAC-60 (3.893 kg/ha) e as linhagens de trigo duro 14 (3.987 kg/ha), 21 (3.908 kg/ha), 25 (3.871 $\mathrm{kg} / \mathrm{ha})$ e $8(3.816 \mathrm{~kg} / \mathrm{ha})$.

As linhagens de trigo duro estudadas, recentemente introduzidas do CIMMYT (Camargo et al., 1993), quando comparadas com cultivares de trigo e de triticale adaptados às condições paulistas, em condição de solo corrigido de Monte Alegre do Sul (V\% entre 48 e $57 \mathrm{e} \mathrm{H}^{+}+\mathrm{Al}^{3+}$ entre 28 e 29 $\mathrm{mmol}_{\mathrm{c}} / \mathrm{dm}^{3}$ ) e de Campinas (V\% entre 43 e 62 e $\mathrm{H}^{+}+\mathrm{Al}^{3+}$ entre 37 e $\left.39 \mathrm{mmol}_{\mathrm{c}} / \mathrm{dm}^{3}\right)$ - Quadro 1 com irrigação por aspersão, mostraram elevado potencial de produção, indicando a possibilidade de, num futuro próximo, campos comerciais com essa espécie produzirem grãos para obtenção de farinha (semolina) adequada à elaboração de macarrão.

Essa possibilidade não se concretizaria se os genótipos de trigo duro fossem cultivados em solos ácidos ou naqueles somente com a camada arável corrigida, em condição de sequeiro, pois essa espécie tem mostrado elevada sensibilidade à toxicidade de alumínio (Camargo et al., 1992, 1993).

Os graus médios de infecção de ferrugem-da-folha, ferrugem-do-colmo, oídio e mancha-da- folha, nos genótipos de cada experimento em 1987-88, encontram-se no quadro 5.

Em relação à ferrugem-da-folha, destacaram-se, quanto à resistência em planta adulta, a linhagem de trigo duro 19, que se mostrou imunc, e a 22 , que apresentou apenas algumas pústulas (tS).

As demais linhagens e o cultivar de triticale Álamos revelaram-se resistentes, com graus máximos de infecção entre 5 e $10 S$, já os cultivares Seri-82 e IAC-24, com reações máximas de $40 \mathrm{~S}$, o IAC-29, com 30 S e O IAC-60 com $20 \mathrm{~S}$ foram os genótipos mais suscetíveis.

Não houve, no período, condições naturais favoráveis para infecção do agente causal da ferrugem-do-colmo. Somente no ensaio de Campinas (1987), detectou-se sua ocorrência; as linhagens de trigo duro $5,6,10,16,18,19$ e 22 e o cultivar de triticale Álamos foram imunes ao patógeno, e 
Quadro 6. Altura média das plantas $\left({ }^{1}\right)$, porcentagem média de acamamento e ciclo da emergência ao florescimento das linhagens de trigo duro (Triticum durum L.) em comparação com cultivares de trigo (Triticum aestivum L.) e de triticale (X Triticosecale Wittmack), em ensaios desenvolvidos em condição de irrigação, na Estação Experimental de Monte Alegre do Sul (1986-89) e no Centro Experimental de Campinas (1987-88)

Altura das plantas

Ciclo $\left(^{2}\right)$

Emerg.-Flor.

Trigo duro (Triticum durum L.)

1. ROK " $S$ "

2. BOY "S"

3. TRA "S"

4. SWAN "S"

5. SCO "S"/MEXI "S"

6. DACK "S"/GDOVZ 394

7. BOY "S"/YAV "S"

8. GEDIZ "S"/YAV "S"

9. CARCOMUM "S"

10. MEMO "S"/MEXI 75

11. MEMO "S"/MEXI 75

12. MEMO "S"/MEXI 75

13. MEMO "S"/GOO "S"

14. $61150 / \mathrm{LDS} / / \mathrm{GLL}$ "S"/3/GR "S"/4/MEXI "S"/5/S15-CR "S"

15. USDA 580/3/CIT "S"/BA $81 / / \mathrm{FG}$ "S"/4/GOO "S"

16. USDA 595/DACK "S"//ROK "S"

17. GS "S"/TC 60//MEXI "S"/3/

GEDIZ "S"/4/CTA"S"

18. RAIL "S"

19. MINDUM/KIF "S"//SAPI "S"

20. SCO "S"/RABI "S"//MEXI 75

21. BOY "S"/CIT 71/5/CR "S"/GS "S"//MT "S"/3/TILO "S"/4/MEMO "S"

22. GTA "S" /DURUM 69

23. MEXI 75

24. YAV 79

25. GA "S"

$83 \mathrm{bc}$
$83 \mathrm{bc}$
$83 \mathrm{bc}$
$75 \mathrm{c}$

24

32

8

20

$76 \mathrm{c}$

$80 \mathrm{bc}$

$80 \mathrm{bc}$

$81 \mathrm{bc}$

$77 \mathrm{c}$

$75 \mathrm{c}$

$76 \mathrm{c}$

$76 \mathrm{c}$

$79 \mathrm{bc}$

$83 b c$

$74 \mathrm{c}$

12

16

$73 \mathrm{c}$

$80 \mathrm{bc}$

$75 \mathrm{c}$

$79 \mathrm{bc}$

$74 c$

16

8

12

12

$76 \mathrm{c}$

20

$76 c$

16

81 bc

24

$81 b c$

20

$80 \mathrm{bc}$
Precoce

Médio

Médio

Médio

Tardio

Médio

Tardio

Tardio

Tardio

Médio

Tardio

Tardio

Médio

Médio

Tardio

Médio

Tardio

Médio

Tardio

Tardio

Médio

Tardio

Precoce

Médio

Tardio

Continua 
Quadro 6. Conclusão

\begin{tabular}{lccc} 
Linhagens e/ou cultivares & $\begin{array}{c}\text { Altura das } \\
\text { plantas }\end{array}$ & Acamamento & $\frac{\text { Ciclo }\left(^{2}\right)}{\text { Emerg.-Flor. }}$ \\
\hline
\end{tabular}

\section{Triticale (X Triticosecale Wittmack)}

26. ÁlAMOS

$95 \mathrm{a}$

24

Precoce

Trigo (Triticum aestivum L.)

\begin{tabular}{llll} 
27. Seri-82 & $81 \mathrm{bc}$ & 28 & Médio \\
28. IAC-24 & $88 \mathrm{ab}$ & 20 & Médio \\
29. IAC-60 & $96 \mathrm{a}$ & 24 & Precoce \\
30. IAC-29 & $77 \mathrm{c}$ & 12 & Precoce \\
\hline F (Genótipos) & $6,73 *$ & \\
d.m.s. (Tukey a 5\%) & 11 & \\
CV \% & 6,52 & \\
\hline
\end{tabular}

* Significativo ao nível de 5\%. ( ${ }^{1}$ ) Médias seguidas de, pelo menos, uma letra em comum, não diferem pelo teste de Tukey. $\left.{ }^{2}\right)$ Precoce: 61 a 70 dias da emergência ao florescimento; médio: 71 a 80 dias; tardio: $81-90$ dias, e muito tardio: 91 a 100 dias.

as linhagens $3,13,20$ e 21 apresentaram maior suscetibilidade, com graus de infecção máximo variando entre $40 \mathrm{~S}$ e $60 \mathrm{~S}$.

Em relação ao oídio, destacou-se quanto à resistência em planta adulta, nos ensaios de Campinas (1987-88), a linhagem de trigo duro 11 e os cultivares Álamos, de triticale, e IAC-29, de trigo, que se mostraram imunes. Consideraram-se as linhagens de trigo duro 4, 6, 9, 10, 13 e 18 resistentes por mostrar uma porcentagem de área foliar infectada entre 1 e $5 \%$.

Os demais genótipos mostraram-se moderadamente resistentes (porcentagem de área foliar infectada de 6 a 20\%), com exceção da linhagem de trigo duro 7 , considerada suscetível ao patógeno (40\% de área foliar infectada).

A linhagem de trigo duro 6 apresentou-se como moderadamente resistente aos agentes causais das manchas foliares, em estádio de planta adulta, com grau máximo de infecção 20. As linhagens 3,7 , 8 e 24 foram consideradas suscetíveis $\mathrm{c}$ os demais genótipos, altamente suscetíveis.

As alturas médias das plantas, as porcentagens médias de acamamento e os ciclos médios da emergência ao florescimento dos genótipos dos ensaios dos dois locais paulistas em 1986-89 estão no quadro 6.

O cultivar de triticale Álamos e o de trigo IAC-60 , exibiram as plantas mais altas, diferindo dos demais genótipos, com exceção do 'IAC-24', pelo teste de Tukey, ao nível de 5\%. As linhagens 4, $5,9,10,11,12,15,16,18,20,21$ e 22 e o 'IAC-29' apresentaram as plantas mais baixas, sendo, portanto, boas fontes de nanismo para cruzamentos.

As linhagens de trigo duro 2 e 25 apresentaram uma porcentagem média de plantas acamadas: 32 e 36 respectivamente. 
As linhagens de trigo duro $3,6,7,10$ e 18 exibiram menor porcentagem de plantas acamadas $(0$ a $8 \%$ ), estando, portanto, entre aquelas com potencial de cultivo em condição de irrigação por aspersão.

Os cultivares de trigo IAC-60 e IAC-29, o de triticale 'Álamos', a linhagem 14 apresentaram-se como precoces, com ciclo médio da emergência ao florescimento entre 61 e 70 dias. Consideraram-se os demais genótipos como de ciclo médio ou tardio.

\section{CONCLUSÕES}

1. As linhagens de trigo duro 14 (61150/ Leeds//Gallo "S"/3/Garza "S"/4/Mexicali "S"/5/S15-Crane "S"), 21 (Boyeros "S"/Cocorit-71/5/Crane "S"/Ganso "S"//Marte "S"/3/Tildillo "S"/4/Memo "S"), 25 (Gallareta "S") e 8 (Gediz "S"/Yavaros "S"), resistentes às ferrugens-do-colmo (com exceção da linhagem 21) e da-folha, moderadamente resistentes ao oídio, suscetíveis à mancha foliar, de porte baixo a médio, destacaram-se quanto à produção de grãos, em solos com baixa acidez, com irrigação por aspersão, não diferindo do cultivar de trigo comum IAC-60, o mais cultivado atualmente no Estado de São Paulo, e do cultivar de triticale Álamos.

2. A linhagem de trigo duro 19 (Mindum/Kingfisher "S"//Sandpiper) apresentou-se ao mesmo tempo imune aos agentes causais das ferrugens-do-colmo e da-folhă e moderadamente resistente ao do oídio.

3. O cultivar de triticale Álamos e o de trigo comum IAC-29 exibiram imunidade ao agente causal do oídio.

4. Todos os genótipos avaliados foram suscetíveis aos patógenos causadores das manchas foliares, com exceção da linhagem 6 (Dackiye/Gerardo Vezio 394), moderadamente resistente.

\section{REFERÊNCIAS BIBLIOGRÁFICAS}

CAMARGO, C.E. de O.; CAMARGO, C.R. de O.; FELÍCIO, J.C.; FERREIRA FILHO, A.W.P.; SANTOS, R.R. dos \& DECOT, G. Avaliação das características agronômicas e tecnológicas de genótipos de trigo duro, trigo e triticale. Campinas, Instituto Agronômico, 1993. 27p. (Boletim científico, 29)

CAMARGO, C.E. de O.; FELÍCIO, J.C.; FERREIRA FILHO, A.W.P.; BARROS, B. de C.; FREITAS, J.G. de; PETTINELli JUNIOR, A.; GALLO, P.B. \& KANTHACK, R.A.D. Melhoramento do trigo: XXV. Avaliação de genótipos oriundos de populações híbridas introduzidas de Oregon (EUA) no Estado de São Paulo. Bragantia, Campinas, 50(2):225-246, $199 \mathrm{I}$.

CAMARGO, C.E. de O.; FELÍCIO, J.C.; FERREIRA FILHO, A.W.P.; BARROS, B. de C.; PETTINELLI JUNIOR, A. \& SANTOS, R.R. dos. Trigo duro, trigo comum e triticale: avaliação de linhagens em condiçōes de irrigação e de soluções nutritivas. Arquivos do Instituto Biológico, São Paulo, 60(1/2):38-47, 1995.

CAMARGO, C.E. de O., SANTOS R.R. dos \& PETTINELLI JUNIOR, A. Trigo duro: tolerância à toxicidade do alumínio em soluções nutritivas e no solo. Bragantia, Campinas 51(1):69-76, 1992.

HANSON, J.; BORLAUG, N.E. \& ANDERSON, R.G. Wheat in the third world. Boulder, Westview Press, 1982. $174 \mathrm{p}$.

MEHTA Y.R. Doenças do trigo e seu controle. São Paulo, Agronômica Ceres, 1978. 190p. (Ceres, 20)

PIMENTEL GOMES, F. Curso de estatística experimental. 11.ed. São Paulo, Nobel, 1985. 466p.

SÃO PAULO. Secretaria de Agricultura e Abastecimento. Relatório do Acordo entre a SAA, através do Instituto Agronômico, e as Cooperativas Rurais do Vale do Paranapanema, Campinas, 1984-1992. 9v.

SCHRAMM, W.; FULCO, W.S.; SOARES, M.H.G. \& ALMEIDA, A.M.P. Resistência de cultivares de trigo em experimentação ou cultivo no Rio Grande do Sul, às principais doenças fúngicas. Agronomia Sulriograndense, Porto Alegre, 10(1):31-52, 1974. 